\section{Seizures due to hyponatremia following poly- ethylene glycol preparation; a report of two cases}

Colonoscopic screening and adenoma removal have been reported to reduce deaths from colorectal cancer $[1,2]$. Proper bowel preparation is needed for adequate visualization of the colonic mucosa [3]. Issues concerning the safety of oral sodium phosphate have been raised, so guidelines recommend the use of polyethylene glycol (PEG) $[3,4]$. It has been reported that using PEG for bowel cleansing prior to colonoscopy does not cause any electrolyte disturbances [5]. However, there have also been reports of serious adverse events related to PEG use $[4,6]$.
We report here on two women who were admitted with generalized tonic-clonic seizures induced by precolonoscopic PEG preparation. Their pertinent clinical and laboratory data are shown under patients $\# 1$ and \#2 in $\bullet$ Table 1. They were treated with intravenous sodium solutions; as their sodium levels recovered, they both showed complete neurologic recovery. Follow-up visits showed normal sodium levels without neurologic deficits.

Along with the characteristics of our patients, the clinical findings of the previously reported cases of hyponatremia due to PEG are also shown in $\square$ Table 1. All of the patients were aged over 50, with four being over 60. Patient \#3 had pre-existing end-stage renal disease and patient \#4 was taking thiazide diuretics, which would have impaired her ability to excrete water [7]. Patient \#5 was similar to patient \#1 in that she showed normal renal, thyroid, and adrenal function, but had ingested $4 \mathrm{~L}$ of fluids in addition to the 3 L of PEG [8]. Nonosmolar antidiuretic hormone stimulation combined with old age and a large volume of fluid most likely caused her hyponatremia. The final patient had been taking serotonin reuptake inhibitors, had inadequate thyroid replacement, and was aged over 70 , which would have further aggravated her hyponatremia [9].

Our two patients developed hyponatremia even with the relatively safe laxative

Table 1 A summary of the clinical findings in our two patients (\#1 and \#2) and other patients reported in the literature with polyethylene glycol (PEG)-related hyponatremia.

\begin{tabular}{|c|c|c|c|c|c|c|}
\hline Patient number & 1 & 2 & $3[7]$ & $4[7]$ & $5[8]$ & 6 [9] \\
\hline Age & 70 & 65 & 51 & 62 & 59 & 73 \\
\hline Sex & Female & Female & Male & Female & Female & Female \\
\hline $\begin{array}{l}\text { Past history/ } \\
\text { underlying disease }\end{array}$ & $\begin{array}{l}\text { Hypertension, } \\
\text { osteoporosis, mild } \\
\text { stenosis of internal } \\
\text { carotid artery }\end{array}$ & $\begin{array}{l}\text { Breast cancer, } \\
\text { total thyroidec- } \\
\text { tomy and radio- } \\
\text { iodine therapy }\end{array}$ & $\begin{array}{l}\text { Diabetes, end- } \\
\text { stage renal failure }\end{array}$ & $\begin{array}{l}\text { Hypertension, } \\
\text { hyperlipidemia }\end{array}$ & $\begin{array}{l}\text { Hysterectomy with } \\
\text { oophorectomy }\end{array}$ & $\begin{array}{l}\text { Hypothyroidism, } \\
\text { depression }\end{array}$ \\
\hline Prescription drugs & $\begin{array}{l}\text { Amlodipine, } \\
\text { ibandronic acid, } \\
\text { clopidogrel }\end{array}$ & Levothyroxine & $\begin{array}{l}\text { Amlodipine, ateno- } \\
\text { lol, furosemide, } \\
\text { calcium acetate, } \\
\text { omeprazole }\end{array}$ & Thiazide & Estradiol, aspirin & $\begin{array}{l}\text { Levothyroxine, } \\
\text { citalopram }\end{array}$ \\
\hline Preparation methods & $\begin{array}{l}\text { 4L PEG and } \\
3 \text { L clear water }\end{array}$ & 4L PEG & $\mathrm{N} / \mathrm{A}$ & 4L PEG & $\begin{array}{l}3 \mathrm{~L} \text { PEG and } \\
4 \mathrm{~L} \text { weak tea }\end{array}$ & $\begin{array}{l}255 \mathrm{~g} \text { PEG and } 64 \\
\text { ounces Gatorade }\end{array}$ \\
\hline Clinical presentation & Seizure & Seizure & $\begin{array}{l}\text { Emesis, idioven- } \\
\text { tricular rhythm, } \\
\text { cardiac arrest }\end{array}$ & Seizure & Confusion & Seizure \\
\hline Blood pressure, $\mathrm{mmHg}$ & $190 / 100$ & $156 / 85$ & $167 / 78$ & $130 / 90$ & $110 / 70$ & $\begin{array}{l}\text { Within normal } \\
\text { range }\end{array}$ \\
\hline Pulse, beats per min & 84 & 86 & 103 & 90 & 60 & $\begin{array}{l}\text { Within normal } \\
\text { range }\end{array}$ \\
\hline \multicolumn{7}{|l|}{ Sodium, mmol/L } \\
\hline Baseline & 140 & 144 & 138 & 138 & $\mathrm{~N} / \mathrm{A}$ & $\mathrm{N} / \mathrm{A}$ \\
\hline Lowest & 110 & 127 & 122 & 116 & 120 & 117 \\
\hline Post-treatment & 138 & 141 & $\mathrm{~N} / \mathrm{A}$ & 130 & 138 & 131 \\
\hline Potassium, mmol/L & 3.4 & 4.3 & 5.1 & 3.9 & 4.6 & 3.3 \\
\hline Chloride, $\mathrm{mmol} / \mathrm{L}$ & 72 & 104 & 94 & 79 & $N / A$ & 79 \\
\hline Bicarbonate, $\mathrm{mmol} / \mathrm{L}$ & 17.3 & 17.3 & 20 & 26 & 17.2 & 21 \\
\hline Urea, mg/dL & 11.8 & 14.6 & 24.3 & 2.5 & $N / A$ & 6 \\
\hline Creatinine, $\mathrm{mg} / \mathrm{dL}$ & 0.67 & 0.71 & 7.7 & 0.6 & 0.9 & 0.6 \\
\hline Glucose, mg/dL & 148 & 235 & 95.5 & N/A & 93 & $\mathrm{~N} / \mathrm{A}$ \\
\hline Brain CT/MRI findings & No abnormalities & No abnormalities & Not done & Cerebral edema & No abnormalities & Not done \\
\hline Treatment & IV $3 \%$ saline & IV normal saline & None & IV $3 \%$ saline & IV normal saline & $\begin{array}{l}\text { IV } 2 \% \text { saline, then } \\
\mathrm{NaCl} \text { tablets \& } \\
\text { water restriction }\end{array}$ \\
\hline Outcome & Complete recovery & $\begin{array}{l}\text { Complete } \\
\text { recovery }\end{array}$ & Death & $\begin{array}{l}\text { Complete } \\
\text { recovery }\end{array}$ & $\begin{array}{l}\text { Complete } \\
\text { recovery }\end{array}$ & $\begin{array}{l}\text { Complete } \\
\text { recovery }\end{array}$ \\
\hline
\end{tabular}

$\mathrm{N} / \mathrm{A}$, not available; IV, intravenous; $\mathrm{NaCl}$, sodium chloride. 
PEG, which raises safety issues that should be carefully considered when instituting colonoscopic procedures.

\section{Endoscopy_UCTN_Code_TTT_1AO_2AN}

Competing interests: None

M. K. Baeg', J. M. Park', S. H. Ko², G. J. Min', K. J. Lee', J. H. Yang', C. H. Lim', S. W. Kim ${ }^{1}$, M.-G. Choi ${ }^{1}$

${ }^{1}$ Division of Gastroenterology, Department of Internal Medicine, College of Medicine, The Catholic University of Korea, Seoul, Korea

${ }^{2}$ Division of Endocrinology, Department of Internal Medicine, College of Medicine, The Catholic University of Korea, Seoul, Korea

\section{References}

1 Lieberman D. Colorectal cancer screening: practice guidelines. Dig Dis 2012; 30 (Suppl. 02): $34-38$

2 Zauber AG, Winawer SJ, O'Brien MJ et al. Colonoscopic polypectomy and long-term prevention of colorectal-cancer deaths. NEJM 2012; 366: 687-696

3 Hassan C, Bretthauer M, Kaminski MF et al. Bowel preparation for colonoscopy: European Society of Gastrointestinal Endoscopy (ESGE) guideline. Endoscopy 2013; 45: $142-150$

4 Belsey J, Epstein O, Heresbach D. Systematic review: adverse event reports for oral sodium phosphate and polyethylene glycol. Aliment Pharmacol Ther 2009; 29: 15-28

5 Cohen $C D$, Keuneke C, Schiemann $U$ et al. Hyponatraemia as a complication of colonoscopy. Lancet 2001; 357: $282-283$

6 Hasan AG, Brown WR. Colonic cleansing for colonoscopy: a risk to be taken seriously. Gastrointest Endosc 2011; 73: 616-618

7 Ayus JC, Levine R, Arieff AI. Fatal dysnatraemia caused by elective colonoscopy. BMJ 2003; 326: 382 - 384

8 Schroppel B, Segerer S, Keuneke C et al. Hyponatremic encephalopathy after preparation for colonoscopy. Gastrointest Endosc 2001; 53: 527-529

9 Nagler J, Poppers D, Turetz M. Severe hyponatremia and seizure following a polyethylene glycol-based bowel preparation for colonoscopy. J Clin Gastroenterol 2006; 40: 558-559
Bibliography

Dol http://dx.doi.org/

10.1055/s-0033-1344568

Endoscopy 2013; 45: E269-E270

(c) Georg Thieme Verlag KG

Stuttgart · New York

ISSN 0013-726X

\section{Corresponding author}

\section{J. M. Park, MD}

Department of Internal Medicine, College of Medicine

The Catholic University of Korea, \# 505, Banpo-Dong

Seocho-Gu

Seoul, 137-701

Korea

Fax: +82-2-22582055

parkjerry@catholic.ac.kr 